\title{
On the stability of atom chip interferometers
}

\author{
Matthew B. Squires, ${ }^{1}$ Brian Kasch, ${ }^{1}$ Spencer E. Olson, ${ }^{1}$ and James A. Stickney ${ }^{2}$ \\ ${ }^{1}$ Air Force Research Laboratory, Kirtland AFB, New Mexico 87117, USA \\ ${ }^{2}$ Space Dynamics Laboratory, Bedford, Massachusetts 01730, USA
}

\begin{abstract}
We calculate the sensitivity to changes in temperature and current for a resonant atom interferometer device based on oscillations of a cold, magnetically trapped cloud in a two wire harmonic trap. The sensitivity of the trap frequency and area enclosed are estimated for typical trap parameters. Based on this analysis, the frequency and area enclosed are both more sensitive to the stability of the current creating the magnetic field than the thermal expansion of the substrate. Current experimental progress measuring the frequency stability of current atom chip devices is outlined.
\end{abstract}

\section{INTRODUCTION}

Atom chips have great versatility in producing a wide variety of potentials for trapping and manipulating cold neutral atoms [1-3], which has led to various demonstrations of cold atom sensors [4-7]. These potentials are generally magnetic and manipulate the atoms by interaction with the atomic magnetic dipole moment. The stability of any atom chip device or sensors depends on the stability of the magnetic field, which in turn depends on the stability of the sources generating the magnetic field. The result of the errors will be a frequency shift of the free oscillation for atoms in the magnetic trap. While there are many potential sources of error, we will consider two that are particularly relevant for a device based on a harmonic trap [8]: the thermal expansion of the atom chip and the stability of the current used to generate the magnetic field of the potential.

This paper first discusses the effect of frequency shifts on the bias stability of an ideal resonant confined atom interferometer. Following this, a derivation of the frequency shifts to a tunable harmonic trap due to thermal expansion and the current stability of the power supply will be given. We will briefly present recent experimental results of the frequency stability of cold neutral atoms oscillating in a harmonic magnetic potential.

\section{STABILITY OF A RESONANT GYROSCOPE}

The Sagnac phase shift is

$$
\Delta \phi=\frac{4 \pi N \Omega A}{\lambda v}
$$

where the vector quantities of $\Omega$ and $A$ are dropped by assuming the rotation is maximally sensed, $N$ is the number of times the atoms traverse the area, $\lambda$ is the wavelength of the particle, and $\Delta \phi$ is the induced phase shift in the interferometer.

For cold atoms, the wavelength is determined using the de Broglie wavelength $\lambda=h / p=h /(m v)$ so the denominator simplifies to $h / m$.

As seen in Fig. 1, assume that the atoms are executing harmonic motion in both directions. The atoms are oscillating in a magnetic trap formed by wires under the

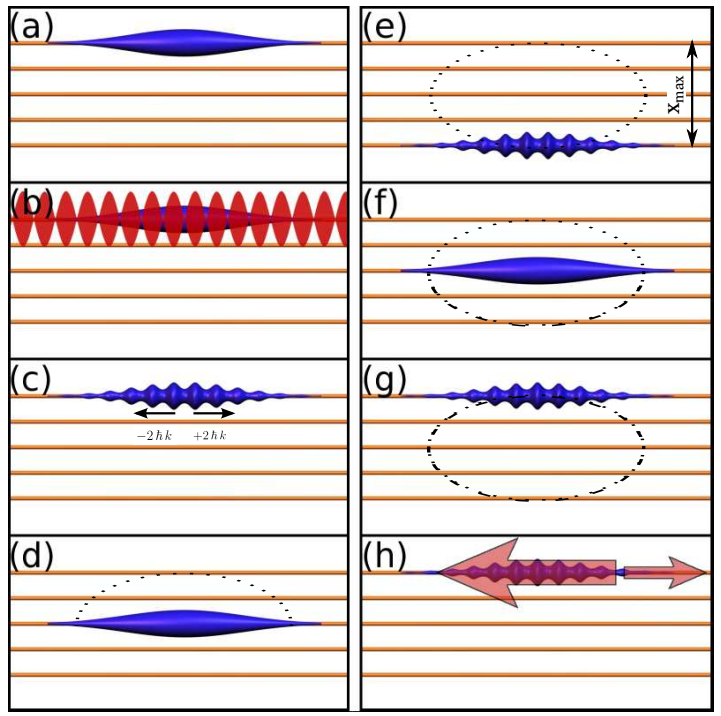

FIG. 1. A schematic of an interferometer cycle. (a) The cold gas is loaded into a cigar shaped trap, created with the upper most horizontal wire (b) The atomic cloud is illuminated with a standing wave laser field imparting a $\pm 2 \hbar k$ momentum kick. (c) After the laser pulse, a density modulation appears across the cloud. (d) The density modulation disappears and the trap is moved downwards by cycling the current in the wires. The dashed line denotes the approximate classical path of individual atoms in the cloud. (e) The trap is above the bottom wire at a half period of the parallel trap and the density modulation reappears across the cloud. The atoms have been maximally translated by the length of the atom chip $x_{\max }$. (f) The trap is moved upward and the density modulation disappears. (g) The trap reaches the top wire at one trap period of the parallel trap and the density modulation reappears for a second time. (h) A probe pulse is reflected off of the cloud.

surface (not shown) but may be formed by the wire pattern shown in Fig. 2 which is the basis for the atom chip currently in use. The parallel wires shown in Fig. 1 are energized sequentially in order to translate atoms perpendicularly to the direction of oscillation. Thus, the atoms enclose an area in order to be sensitive to rotations. The 
equation of motion in each direction is

$$
\begin{array}{r}
x_{\|}=\frac{2 \hbar k}{m \omega} \sin (\omega t) \\
x_{\perp}=x_{\max } \sin (\omega t)
\end{array}
$$

where $k$ is the wavenumber of the standing wave and $\omega$ is the period of oscillation. The period of oscillation is the same for both axes so the device will be resonant. With that equation of motion, the area enclosed is

$$
A=\pi x_{\max } \frac{2 \hbar k}{m \omega} .
$$

In the end, we want to know how temperature variations lead to the change in the area of the interferometer. The area enclosed is

$$
\begin{aligned}
A_{\text {enc }} & =\frac{h k}{m} \frac{x_{\max }}{\omega} \\
& =\frac{h k}{m} \frac{\left(x_{\max }+\delta_{x}\right)}{\left(\omega+\delta_{\omega}\right)}
\end{aligned}
$$

where $x_{0}$ and $\omega_{0}$ are the designed parameters and the $\delta$ s are the variations due to the temperature shifts. Expanding as a series and only keeping terms that are first order in $\delta$

$$
\begin{aligned}
A_{e n c} & \approx \frac{h k x_{0}}{m \omega_{0}}+\frac{h k}{m \omega}\left(\delta_{x}-x_{\max } \frac{\delta_{\omega}}{\omega}\right) \\
& =A_{0}+\delta A .
\end{aligned}
$$

This change in area becomes an error in the measured rotation rate. Following a similar derivation

$$
\begin{aligned}
\Omega+\delta \Omega & =\frac{h}{S N R 4 \pi N\left(A_{0}+\delta_{A}\right)} \\
& \approx \frac{h}{S N R 4 \pi N A_{0}}\left(1-\delta_{A} / A_{0}\right)
\end{aligned}
$$

Therefore the error in the rotational rate is

$$
\delta \Omega=-\frac{\delta_{A}}{A_{0}} \Omega
$$

\section{VARIATIONS TO $A_{\text {enc }}$}

For the system described above, the two explicit sources of variations in $A_{\text {enc }}$ are the change in size of the atom chip perpendicular to the oscillation direction or along $x_{\max }$ and the change in frequency of oscillation. It is assumed that there will be other sources of error that will need to be analyzed, such as the frequency characteristics of the current noise, however only the temperature dependence of the atom chips and DC current stability are discussed in this paper.

\section{A. Single wire pair potential}

Assuming the magnetic field is generated by symmetric pairs of wires or coils, the magnetic field can be expressed as an even polynomial, i.e. $B(x)=c_{0}+c_{2} x^{2}+c_{4} x^{4}+\cdots$, where each term includes the overall magnetic field magnitude. The frequency of a particle in a near harmonic magnetic field $\left(c_{2} \gg c_{i}\right.$ where $\left.i \geq 4\right)$ is then given by $\omega=\sqrt{2 \mu_{B} c_{2} / m}$, where $\mu_{B}$ is the dipole moment of the particle and $m$ is the mass of the trapped particle.

Similar to Helmholtz or anti-Helmholtz coils, the spacing of the wires affects the various contributions of the expansion coefficients to the magnetic field or the relative strength of the coefficients $c_{\mathrm{i}}$. While a single wire pair may be used to minimize a single term in the magnetic field expansion, as is common for designing a Helmholtz magnetic coil, multiple wire pairs may be used to provide control over multiple terms in the expansion and to provide dynamic tuning of the terms [8]. This is the function of the multiple wire pairs shown schematically in Fig. 2.

For simplicity only the harmonic contribution of a single wire pair will be considered. The harmonic term is

$$
c_{2}=\frac{I \mu_{0}}{2 \pi} \frac{W^{4}+4 W^{2} Z^{2}-Z^{4}}{Z\left(W^{2}+Z^{2}\right)}
$$

where $\mu_{0}$ is the magnetic constant, $W$ is the half spacing between the wires, and $Z$ is the distance from the center of the wires to the position of the atoms. The frequency changes as the wire spacing changes due to the thermal expansion of the atom chip.

\section{B. $\delta_{x}$ variations}

The change in size of the trapped atom device is $\delta_{x}(T)=x_{\max } c_{\mathrm{CTE}} \Delta T$ where $c_{\mathrm{CTE}}$ is the coefficient of thermal expansion of the atom chip and $\Delta T=T-T_{0}$ is the temperature variation around an equilibrium temperature $T_{0}$. For typical atom chip configurations, the metal layer is much thinner than the substrate such that the thermal expansion is largely determined by the thermal expansion of the substrate.

\section{C. $\delta_{\omega}$ variations}

The change in frequency of the trap depends on the current that creates that trap and the thermal expansion of the atom chip which determines the position of the wires. The change in frequency is

$$
\begin{aligned}
\delta_{\omega}(T, I) & =\frac{\partial \omega}{\partial T} \Delta T+\frac{\partial \omega}{\partial I} \Delta I \\
& =\delta_{\omega}(\Delta T)+\delta_{\omega}(\Delta I)
\end{aligned}
$$

where $\Delta T$ is the same as the $\delta_{x}$ variations and $\Delta I=I-I_{0}$ where $I_{0}$ is the set current for a given magnetic trap $\omega$. 
The change in frequency as a function of wire or coil spacing $W$ is

$$
\begin{aligned}
\delta_{\omega}(\Delta T) & =\frac{d \omega}{d c_{2}} \frac{d c_{2}}{d W} \frac{d W}{d T} \Delta T \\
& =\frac{d \omega}{d W} W c_{\mathrm{CTE}} \Delta T,
\end{aligned}
$$

where $\frac{d W}{d T}=W c_{\mathrm{CTE}}$.

Similarly, the frequency stability as a function of the current stability is

$$
\begin{aligned}
\delta_{\omega}(\Delta I) & =\frac{d \omega}{d c_{2}} \frac{d c_{2}}{d I} \Delta I \\
& =\frac{d \omega}{d I} \Delta I .
\end{aligned}
$$

\section{Total contributions}

The combined variation to $A_{\text {enc }}$ is

$\delta_{A}=\frac{h k}{m \omega}\left(x_{\max } c_{\mathrm{CTE}}\left(1-\frac{W}{\omega} \frac{d \omega}{d W}\right) \Delta T-\frac{x_{\max }}{\omega} \frac{d \omega}{d I} \Delta I\right)$.

Using the single wire pair potential, the frequency dependence due to the current and wire spacing can be calculated. The frequency change terms in Eqn. 19 are

$$
\frac{1}{\omega} \frac{d \omega}{d I}=\frac{1}{2 I}
$$

and

$$
\frac{W}{\omega} \frac{d \omega}{d W}=-\left(\frac{7 W^{2} Z^{4}-6 W^{4} Z^{2}-W^{6}}{Z^{6}-3 W^{2} Z^{4}-5 W^{4} Z^{2}-W^{6}}\right) .
$$

The relative order of magnitude variation from variations in temperature and current can be estimated using order of magnitude estimates for each of the terms for typical atom chip parameters. For a harmonic trap the $4^{\text {th }}$ order contribution is canceled when $Z / W \approx 0.75$. Typical atom chip dimensions (i.e. $x_{\max }$ and $W$ ) are on the order of $10^{-2} \mathrm{~m}$ and $c_{\mathrm{CTE}} \approx 10^{-5} \mathrm{~m} / \mathrm{m} / \mathrm{K}$. Typical atom chip currents range between 1-10 A so use $10 \mathrm{~A}$ for these calculations and $\omega \approx 2 \pi 10 \mathrm{~Hz}$. Eqn. 19 becomes

$$
\delta A \approx \frac{h k}{m \omega}\left(1 \times 10^{-7} \Delta T-5 \times 10^{-4} \Delta I\right) .
$$

While $\delta_{A}$ is more sensitive to variations in the current compared to the temperature variations, the typical experimental control over the current is better than typical experimental temperature control, especially for higher power devices like atom chips. Thus, for expected operating conditions, the largest variation is expected to be due to the thermal expansion of the atom chip substrate.

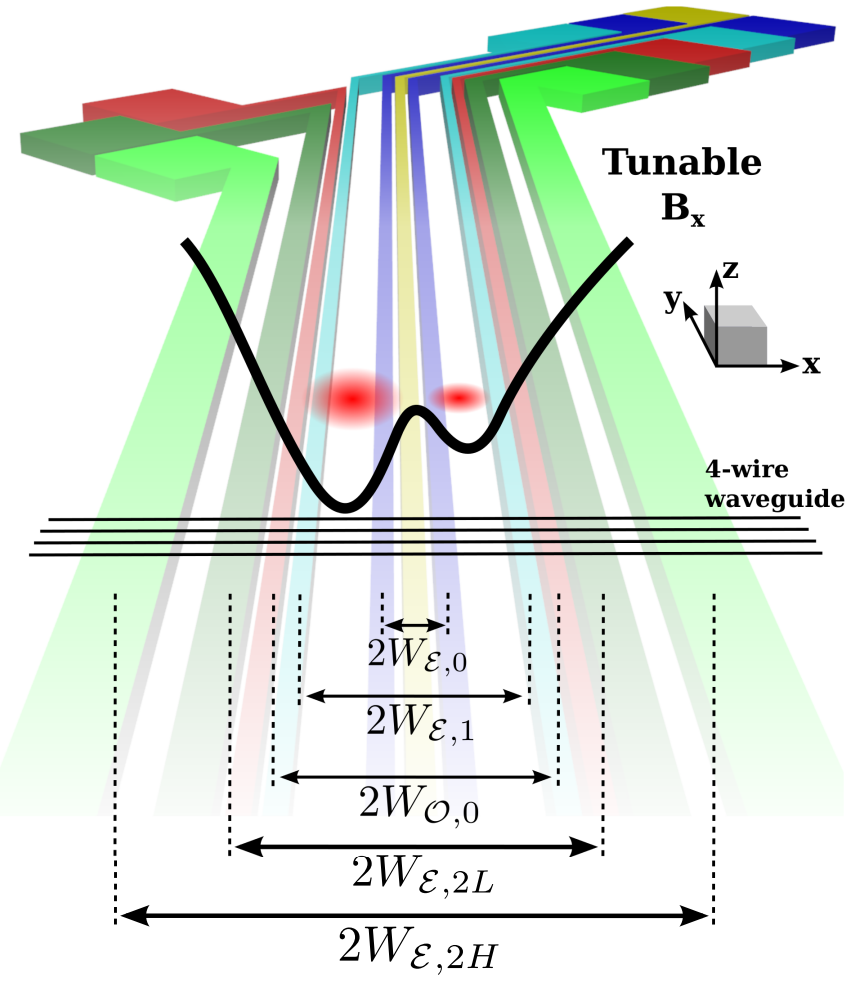

FIG. 2. Multilayer atom chip with tunable control over the magnetic field along the cold atom waveguide formed by crossing wires (shown in black) on the top layer. In the current experimental configuration the four waveguide wires are linked so that the atoms cannot be moved in the transverse direction. This limits the transverse motion of the atoms which improves the ability for testing the stability of the oscillating frequency of atoms in the magnetic trap. Below, even and odd wire pairs, spaced by $2 W_{\mathcal{P}, m}$ give control over the $c_{2}$ and $c_{4}$ and other contributions of the 1D potential.

\section{EXPERIMENTAL STABILITY}

Experimentally, it is common to characterize the stability of a resonant atom chip device by measuring its frequency stability. Fundamental physics questions have been explored by measuring the frequency shift of trapped atoms with a fractional frequency shift of $\lesssim 10^{-4}$ [9]. The change in frequency of a two wire waveguide discussed in this paper is found by rearranging of Eqns. 20, 21 , and 16

$$
\frac{d \omega}{d I}=\frac{\omega}{2 I}
$$

and

$$
\frac{d \omega}{d T}=-c_{\mathrm{CTE}} \omega\left(\frac{7 W^{2} Z^{4}-6 W^{4} Z^{2}-W^{6}}{Z^{6}-3 W^{2} Z^{4}-5 W^{4} Z^{2}-W^{6}}\right) .
$$

Using the same assumptions for the operating parameters of an atom chip device as above, the frequency shifts 
based on the current and temperature stability are

$$
\begin{aligned}
& \frac{d \omega}{d I} \approx 3 \mathrm{~A}^{-1}=180 \mathrm{~Hz} / \mathrm{A} \\
& \frac{d \omega}{d T} \approx-3 \times 10^{-8} \mathrm{~K}^{-1}=-2 \times 10^{-6} \mathrm{~Hz} / \mathrm{K} .
\end{aligned}
$$

Thus the shift in the oscillation frequency as measured in a laboratory experiment is significantly more sensitive to current fluctuations than temperature fluctuations even though for a resonant type gyroscope the temperature and current sensitives are more closely matched. For the current experiment, we are using an atom chip based on the design presented in Ref. [8] and shown in Fig. 2, and are currently in the process of experimentally determining the frequency of this trap. While the analysis shown here is only for a single wire pair, and the atom chip in the experiment uses multiple wire pairs, it is reasonable to expect the two traps will have similar scaling.

The frequency of the trap is experimentally determined by displacing a trapped cloud of laser cooled atoms with linear magnetic field gradient and then turning off the magnetic field gradient. The atoms are allowed to oscillate in the magnetic trap for varying amounts of time and finally absorption imaged. The center of mass of the atom cloud is then calculated by fitting the atom cloud profile to a $2-\mathrm{D}$ Gaussian with a spatial offset. The frequency was extracted by fitting the spatial data to a cosine. One of the contributions to the fitting error are residual imaging artifacts due to imperfections of the glass vacuum chamber. We are in the process of building a new chamber to improve the imaging and fitting error to experimentally determine the stability of trapped atom devices. With the imaging imperfections, the typical fractional frequency fitting error is $\sim 10^{-3}$.

\section{CONCLUSION}

We have derived order of magnitude estimates for temperature and current shifts to the frequency and enclosed areas for atom chip devices. Based on this analysis, atom chip devices are more sensitive to atom chip currents versus shifts due to the thermal expansion of the atom chip substrate. We are in the process of measuring the frequency stability of atoms trapped in a magnetic field trapping potential created by an atom chip and are making improvements to the optical train to improve the error in the frequency measurements.
[1] W. Hänsel, J. Reichel, P. Hommelhoff, and T. W. Hänsch, Phys. Rev. Lett. 86, 608 (2001).

[2] S. Hofferberth, B. Fischer, T. Schumm, J. Schmiedmayer, and I. Lesanovsky, Phys. Rev. A 76, 013401 (2007).

[3] P. Böhi, M. F. Riedel, J. Hoffrogge, J. Reichel, T. W. Hänsch, and P. Treutlein, Nat Phys 5, 592 (2009).

[4] J. D. Carter and J. D. D. Martin, Phys. Rev. A 88, 043429 (2013)

[5] T. Berrada, S. van Frank, R. Bücker, T. Schumm, J.-F. Schaff, and J. Schmiedmayer, Nat Commun 4 (2013), 10.1038/ncomms3077.
[6] H.-C. Chuang, E. A. Salim, V. Vuletic, D. Z. Anderson, and V. M. Bright, Sensors and Actuators A: Physical 165, 101 (2011).

[7] N. P. Robins, P. A. Altin, J. E. Debs, and J. D. Close, Physics Reports 529, 265 (2013).

[8] J. A. Stickney, B. Kasch, E. Imhof, B. R. Kroese, J. A. R. Crow, S. E. Olson, and M. B. Squires, ArXiv e-prints (2014), arXiv:1407.6398 [physics.atom-ph].

[9] J. M. Obrecht, R. J. Wild, M. Antezza, L. Pitaevskii, S. Stringari, and E. A. Cornell, Physical Review Letters 98 (2007), 10.1103/PhysRevLett.98.063201. 